\title{
LAS SEMANAS SOCIALES DE LA IGLESIA PERUANA A LA LUZ DE CARITAS IN VERITATE: EL CASO DE LA PRIMERA SEMANA SOCIAL DE 1959.
}

Fernando Elgegren Reátegui*

\section{RESUMEN}

El Papa Benedicto XVI en su Carta Encíclica Caritas in Veritate deja bien en claro que la vocación cristiana al desarrollo ayuda a buscar la promoción de todos los hombres y de todo el hombre (CIV.18), planteamiento que -lo recuerda el Papa - está sostenido también en las Encíclicas Populorum Progressio de Pablo VI y Centesimus Annus de Juan Pablo II. El autor, al formar parte de la Comisión Episcopal de Acción Social (CEAS) representando a UNIFÉ, participa en la investigación que vienen realizando las universidades católicas de nuestro país sobre la influencia e intervención de la Iglesia en la sociedad peruana durante las últimas décadas. Este artículo relaciona los avances de investigación sobre el estudio de las Semanas Sociales en el Perú con los planteamientos propuestos en la Caritas in veritate.

\section{PALABRAS CLAVE:}

Semanas Sociales en el Perú, Signos de los Tiempos, Acción Social, Acción Católica.

\section{THE SOCIAL WEEKS OF THE PERUVIAN CHURCH UNDER THE LIGHT OF CARITAS IN VERITATE: THE CASE OF THE FIRST SOCIAL WEEK IN 1959.}

\begin{abstract}
Pope Benedict XVI, in his encyclical letter Caritas in Veritate, makes it very clear that "The Christian vocation to development helps to promote the advancement of all men and of the whole man"(CIV.18), an approach that - as the Pope says -is also supported by Pope Paul VI in his encyclical letter Populorum Progressio, and Pope John Paul II's Centesimus Annus. The author, as member representing UNIFE before Peru's Episcopal Commission for Social Action (CEAS), participates in the research performed by the catholic universities of our country on the influence and intervention of the church in Peruvian society in recent decades. This article relates the progress of research on the study of the Social Weeks in Peru with the approaches proposed in the Caritas in veritate.
\end{abstract}

\section{KEY WORDS:}

Social Weeks in Peru, Signs of the Times, Social Action, Catholic Action.

\footnotetext{
* Doctor en Filosofía y Sociólogo por la Pontificia Universidad Católica del Perú. Profesor Principal de la Universidad Femenina del Sagrado Corazón, donde se desempeñó como Jefe del Departamento de Filosofía y Teología y Director (a.i) de la Escuela de Postgrado. Past Presidente de la Sociedad Internacional Santo Tomás de Aquino Sección Perú. Actual vice rector Administrativo.
} 


\section{INTRODUCCIÓN}

A fin de evitar lecturas equívocas sobre el papel de la Iglesia en relación al desarrollo del hombre y de la sociedad, Benedicto XVI insiste a lo largo de la Encíclica que la Iglesia no tiene soluciones técnicas que ofrecer ni pretende mezclarse en la política de los Estados; que sí "tiene una misión de verdad que cumplir en todo tiempo y circunstancia $e$ favor de una sociedad a medida del hombre, de su dignidad y de su vocación" (CV. 9) y que "la fe cristiana se ocupa del desarrollo, no apoyándose en privilegios o posiciones de poder, ni tampoco en los méritos de los cristianos, que ciertamente se han dado y también hoy se dan, junto con sus naturales limitaciones, sino sólo en Cristo, al cual debe remitirse toda vocación auténtica al desarrollo humano integral" y añade que "con las enseñanzas de su Señor, la Iglesia escruta los signos de los tiempos, los interpreta y ofrece al mundo lo que ella posee como propio: una visión global del hombre y de la humanidad" (CV.18).

A la luz de este planteamiento del Papa Benedicto XVI podemos afirmar que la Iglesia en el Perú, por un lado, ha desarrollado un activo papel escrutador de los signos de los tiempos en la realidad peruana y, por otro lado, ha mantenido en diferentes circunstancias y momentos, la fidelidad de su vocación de búsqueda de la promoción del ser humano de manera real y tangible. Concretan estas dos características de la Iglesia en el Perú la organización y realización de las diez Semanas Sociales Nacionales promovidas por el Episcopado Peruano desde 1959 hasta el 2008.

La primera Semana se realizó en Lima en 1959 teniendo como tema las exigencias sociales del catolicismo en el Perú.

La segunda se desarrolló en Arequipa en 1961con el tema sobre la propiedad la misma que tuvo su réplica en Piura y en Ayacucho, en los meses siguientes.
La tercera Semana se realizó en Lima 28 años después de la anterior, como Semana Social del Perú (1989), en la que se trató sobre Estado y Sociedad en el Perú.

En 1991 se llevó a cabo la cuarta Semana Social en Lima con el tema La Iglesia peruana y el mundo del trabajo a los 100 años de la Rerum Novarum, Encíclica del recordado Papa León XIII y que da origen a la Doctrina Social de la Iglesia.

Seis años después, en 1997, se realizó en Lima la quinta Semana con la denominación de Frente al hambre en el Perú, desarrollo solidario.

La sexta fue en 1999 en El Callao sobre Deuda Externa, pobreza desarrollo, en el marco de la preparación inmediata del Año Jubilar.

Después de cuatro años se retoman las Semanas Sociales en el 2003 con la séptima Semana organizada en Lima A los 40 años de la Encíclica Pacem in Terris de Juan XXIII, que plantea a la Iglesia y a todos los hombres y mujeres de buena voluntad construir la paz en base a los cuatro pilares de la verdad, la justicia, el amor y la libertad.

El tema de la octava Semana, fue Construyendo el Bien Común; las alegrías y esperanzas en el Perú de hoy. A los 40 años de la Gaudium et Spes; se desarrolló en Lima en el 2005.

La novena fue en el 2007 en El Callao con el lema "El desarrollo es el nuevo nombre de la paz". A los 40 años de la Populorum Progressio y a los 20 años de la Sollicitudo rei socialis.

La décima y última Semana Social Nacional se realizó en el 2008 en Lima con el tema De Medellín a Aparecida. 40 años de servicio a la evangelización y a la promoción humana en el Perú. 
SEMANAS SOCIALES NACIONALES 1959 - 2008

\begin{tabular}{|c|c|c|c|}
\hline $\mathbf{N}^{\mathbf{0}}$ & Fecha & Lugar & Tema \\
\hline 1 & 1-9/VIII-1959 & Lima & Las exigencias sociales del catolicismo en el Perú \\
\hline 2 & $1-6 /$ VIII-1961 & Arequipa & La propiedad. (Con réplica en Piura en 1963) \\
\hline 3 & $4-9 / I X-1989$ & Lima & Estado y Sociedad en el Perú \\
\hline 4 & 1991 & Lima & $\begin{array}{l}\text { La Iglesia Peruana y el mundo del trabajo a los } 100 \text { años de } \\
\text { la Rerum Novarum }\end{array}$ \\
\hline 5 & 1997 & Lima & Frente al hambre en el Perú, desarrollo solidario \\
\hline 6 & $13-15 / X-1999$ & Callao & $\begin{array}{l}\text { Deuda externa, pobreza y desarrollo. (Como preparación } \\
\text { inmediata del año jubilar). }\end{array}$ \\
\hline 7 & $9-12 / \mathrm{XII}-2003$ & Lima & A los 40 años de la Encíclica Pacem in Terris de Juan XXIII \\
\hline 8 & $18-21 / X-2005$ & Lima & $\begin{array}{l}\text { Construyendo el Bien Común: las alegrías y esperanzas en } \\
\text { el Perú de hoy. A los } 40 \text { años de la Gaudium et Spes. }\end{array}$ \\
\hline 9 & $25-28 / \mathrm{IX}-2007$ & Callao & $\begin{array}{l}\text { El desarrollo es el nuevo nombre de la paz. A los } 40 \text { años } \\
\text { de la Populorum Progressio y a los } 20 \text { años de la Sollicitudo Rei } \\
\text { Socialis }\end{array}$ \\
\hline 10 & $11-14 / \mathrm{XI}-2008$ & Lima & $\begin{array}{l}\text { De Medellín a Aparecida. } 40 \text { años de servicio a la evangeli- } \\
\text { zación y a la promoción humana en el Perú }\end{array}$ \\
\hline
\end{tabular}

Considerando los períodos de los Sumos Pontífices al frente de la Iglesia las dos primeras Semanas (1959 y 1961) se llevaron a cabo durante el Magisterio del Papa Juan XXIII. Las 5 siguientes (de la $3^{\circ}$ a la $7^{\circ}$ ) se realizaron durante el pontificado de Juan Pablo II, mientras que la $8^{\mathrm{a}}, 9^{\mathrm{a}} \mathrm{y}$ $10^{\mathrm{a}}$, tuvieron lugar estando al frente de la Iglesia el actual Pontífice Benedicto XVI.

Como puede apreciarse en el cuadro, entre la primera y segunda Semanas median dos años de distancia y se realizan terminando la década del cincuenta e iniciando la década de los años sesenta (1959-1961); la tercera se realizará luego de 28 años de la segunda, terminando la década de los ochenta (1989); la cuarta aparece como un intento de continuidad de la tercera, toda vez que va en un lapso de dos años de la anterior (1991). La quinta Semana se organiza después de 6 años (1997), con un tema que es más próximo al de la sexta que tendrá lugar dos años más tarde (1999). Pasados cuatro años, las Semanas Sociales se retoman y desarrollan con regularidad: la séptima en el 2003, la octava en el 2005, la novena en el 2007 y la última en el 2008, pasado un año de la anterior.
Cada una de la Semanas Sociales, por los tiempos en que aparecen, los temas desarrollados y las formas de ejecución, se presta como hipótesis de trabajo sobre el papel escrutador de los signos de los tiempos por parte de la Iglesia Peruana así como el de su vocación de promoción del ser humano.

A manera de ejemplo desarrollamos a continuación el análisis e interpretación del papel escrutador de los signos de los tiempos de la Iglesia peruana en el contexto de los inicios de la década de los sesenta, identificando al mismo tiempo el hilo conductor y de sostenimiento que viene a ser la vocación cristiana al desarrollo que busca la promoción humana de todos los hombres $y$ de todo el hombre (CV. 18), que es lo que el Papa refuerza en su Encíclica Caritas in Veritate.

\section{La Iglesia peruana escrutadora de los signos de los tiempos: la primera Carta Pastoral del Episco- pado Peruano y la Primera Semana Social en 1959}

La Primera Semana Social es fruto de la determinación del Episcopado Peruano 
que ordena su realización mediante lo que se conoce como su Primera Carta Social, dada en Lima el 25 de enero, día de la conversión del Apóstol San Pablo, año de 1958, con la firma del Arzobispo de Lima y Primado del Perú y 31 miembros más de la Asamblea Episcopal, integrada por Arzobispos, Obispos, Vicarios, Prefectos y Administradores Apostólicos y Prelados "Nullíus".

La Carta Pastoral de 1958 trata "Sobre algunos aspectos de la cuestión social en el Perú". En esta Carta encontramos la siguiente lectura de la realidad peruana:

- El problema económico social es el más urgente, porque condiciona el desarrollo y la efectividad de las iniciativas apostólicas y afecta la buena disposición para recibir el mensaje evangélico.

- En la economía del Perú "se ha venido gestando una distinción entre zonas que se han desarrollado y otras que permanecen en un nivel rudimentario".

- "Existe una gran presión demográfica sobre la escasa tierra cultivada que debe sustentar una población en su gran mayoría agrícola".

- "La afluencia de riqueza se ha concentrado a menudo en pocas manos ya se trate de la agricultura, la industria y el comercio, y se ha acentuado la contraposición entre los que tienen la riqueza y los que sólo tienen su trabajo".

- La desigualdad refuerza la discriminación: "es un hecho que un gran número de quienes están desprovistos de los bienes suficientes, coincide con la población indígena que vive en los pueblos de las serranías, con obreros ocupados en industrias extractivas o manufactureras, y con jornaleros de algunas zonas agrícolas.....nadie puede negar la gran pobreza, rayana casi en la miseria, y el sentido de depresión en que viven muchos de nuestros hermanos en la Sierra. Nadie puede negar la triste condición de los indígenas emigrados de sus tierras y asalariados en muchas de las haciendas a veces por cortos períodos de tiempo, sin encontrar ningún estímulo que les ayude en la tristeza de su soledad lejos del terruño".

Se podría observar que la realidad nacional, escrutada por los obispos en esta Carta, es breve, genérica, con énfasis en la problemática urbana, obrera, salarial y empresarial, sustentada básicamente en los discursos y escritos del Papa Pío XII, con referencias a las Encíclica Rerum Novarum de León XIII y Quadragesimo Anno de Pío XI. Sin embargo, hay que reconocer que esta Carta, a pesar de la simplicidad en el análisis de la realidad socio-económica, es pionera y certera, al ubicarse en el devenir concreto de la Iglesia y dirigir su mirada a los hombres y su realidad, identificando los problemas sociales prioritarios de la sociedad peruana de la época.

En primer lugar, acierta al señalar que el problema económico es el más urgente y que, vinculado a este, se ha ido generando la brecha entre zonas que se desarrollan frente a otras más atrasadas. Yepes del Castillo (1979), al examinar el desarrollo peruano en las primeras décadas del siglo XX, sostiene que al inicio de la década de los cincuenta se entra a una etapa fundamental "en la cual el capital empieza su desarrollo más profundo en la sociedad peruana" (p.139); y, como nos lo recuerda Julio Cotler (1979) "a partir de 1950 y hasta mediados de la siguiente década, el capital imperialista volvió a irrumpir en el país, favoreciendo el desarrollo de importantes cambios en la estructura de la sociedad peruana" (p.176).

Es la época en la que en el Perú se da inicio al proyecto promovido por la Comisión Económica para América Latina -CEPAL- para lograr el desarrollo 
económico y social mediante el proceso de industrialización por sustitución de importaciones, pregonado y puesto en práctica con antelación en Argentina y Chile. Como da cuenta Parodi (2001) "Antes de 1960, predominaba el modelo liberal primario exportador con una reducida intervención estatal en la economía. A partir de 1960 siguió la estrategia de industrialización por sustitución de importaciones con fuerte presencia del Estado".

La puesta en marcha del proyecto exigía una serie de condiciones de orden estructural y una gran movilización de todas las fuerzas sociales del país para hacerlo viable; se necesitaba modernizar el Estado, ampliar la base económica, nuevos capitales para inversiones en infraestructura, tecnología, insumos, mano de obra capacitada y los especialistas para gerenciar el desarrollo, entre otras. En Lima se crean nuevas universidades en los inicios de la década del 60 con el propósito de responder a los escenarios de la modernización del país que se iban presentando bajo el modelo de sustitución de importaciones. Luis Felipe Angell de Lama (1958), más conocido como "Sofocleto" gana el Premio Nacional de Novela 1958 por su obra trágica "La Tierra Prometida", tierra que no era otra sino Lima en el imaginario del campesino de entonces. Los analistas sociales darán cuenta del proceso en los términos de "modernización frustrada", "desborde popular" o "Leviatán apedreado" al tratar sobre las relaciones Estado y sociedad durante el proceso en cuestión. Por tanto, la Carta del Episcopado Peruano no hace más que reconocer este proceso de transformación y adelantar sus consecuencias.

En segundo lugar, la Carta Pastoral acierta también al señalar sin aspaviento la "presión demográfica", "la concentración de la riqueza", "la desigualdad", "la discriminación" y "la gran pobreza". En efecto, como podemos leer en "Perú: hechos y cifras demográficas" del Concejo Nacional de Población (1985) "en el período de 1940 1965, denominado de 'explosión demográfica, se produjo un acelerado crecimiento natural o vegetativo. La población, que en 1941 bordeaba los 6 millones, llegó a 10 millones en 1961" (p.18).

Asimismo, "ciudadanos, masas y excluidos" como refiere Sinesio López (1991) se entienden en relación a la dinámica del mercado: "El crecimiento del mercado interno, el despegue de la industrialización y el acelerado proceso de urbanización después de los años 50 imprimieron una velocidad creciente a la sociedad de clases y especialmente a la sociedad de masas de informales, subempleados y desempleados". (p.27)

Desde este análisis de Sinesio López se puede entender mejor el sentido del papel escrutador manifiesto en la Carta Pastoral, puesto que no se limita a identificar simplemente los problemas nacionales, sino que la misma Iglesia con el señalamiento de los problemas álgidos de la realidad peruana encuentra su lugar para el discernimiento espiritual que le permite delinear el camino de su propia novedad y lograr la experiencia de estar insertada en la historia. Es por ello que los obispos firmantes de la Carta exigen y ordenan la realización de la Primera Semana Social para "inquietar la conciencia de los cristianos frente a la realidad nacional y promover un amplio y vehemente deseo de investigar a fondo los problemas presentados", finalidad expresada por Monseñor José Dammert Bellido, Secretario General del Episcopado Peruano. El mismo Monseñor explica en las palabras introductorias del libro de la Primera Semana Social (1959) que el tema "Las exigencias sociales del catolicismo en el Perú "se debió a la necesidad de presentar un panorama general de la realidad peruana dejando para futuras Semanas el estudio profundo de los problemas". 
En efecto en la organización de la primera Semana Social se estimó que urgía reforzar el vibrante llamado de los Obispos con el planteamiento sereno y meditado acerca de las clases sociales, las relaciones de trabajo y la promoción cultural (ibid.), cuyo conocimiento y enjuiciamiento, desde un punto de vista cristiano, requerían la mayor atención por parte de "las conciencias católicas".

En el inicio del desarrollo de la Primera Semana, antes de la sustentación de las ponencias, el R.P Felipe Mac Gregor SJ presentó una introducción doctrinal con el tema "Nociones de Comunidad y de Bien Común".

Los temas tratados en ponencias y trabajos de comisiones fueron:

I. Las clases sociales en el Perú: Dr. José Luís Bustamante y Rivero

II. Las relaciones de trabajo: Dr. Jorge del Busto Vargas

III. La promoción cultural: Dr. César Arróspide de la Flor

IV. Orientaciones para la acción de las personas y de las corporaciones privadas: Dr. Luís Echecopar García.

V. Orientaciones para la acción en la vida cívica: Dr. Ernesto Alayza Grundy

VI. Orientaciones para la acción de la Iglesia: Mons. Dammert Bellido.

Todas las exposiciones de la Primera Semana Social inciden en la necesidad de conocer mejor la realidad nacional a pesar de las limitaciones de datos, informaciones e investigaciones.

En el marco de la Carta Pastoral del 58 se destacan algunos planteamientos vertidos por el R.P. Felipe Mac Gregor SJ. y el Dr. José Luís Bustamante y Rivero, los mismos que se encuentran en el libro editado de la Primera Semana Social.

Mac Gregor en su discurso doctrinal e introductorio sostiene que "el bien común es históricamente condicionado; no es lo mismo en la encrucijada peligrosa que vive el Perú hoy, que el que fue en las primeras décadas posteriores a la Independencia o cuando hace cien años se vivía lo que Basadre ha llamado el renacimiento liberal y la ofensiva conservadora". (p.19)

Bustamante y Rivero, con su experiencia de haber sido Presidente del Perú, hace una revisión histórica de la realidad peruana desde el s. XVII al XIX, y puede afirmar: "descubrimos como más característicos dos grupos que poseen perfiles propios, aunque estén recíprocamente muy próximos: el grupo aristocrático y el que posee y ejercita el poder económico. Aristocracia y dinero" (p.40). Puede señalar también que es "una causal más del atraso en el avance socio-económico nuestra recalcitrante inestabilidad política, a través de muchas décadas de golpes de Estado, caudillismo y dictaduras" (p.35). Asimismo, llama la atención sobre el hecho de que "existe un flujo constante de migración nativa sobre la capital de la República. Expresión visible de este fenómeno es la formación de las barriadas populares en casi todas las ciudades importantes del país, en las cuales predomina el elemento indígena. De más está decir que casi sin excepción, el nivel de vida de estas gentes es verdaderamente inhumano" (p.55).

Bustamante y Rivero, examina también a grandes rasgos la misión y la acción de la Iglesia en el campo de las clases sociales, destacando el fomento de obras de caridad "en la cual realizan ejemplar y sacrificada tarea a favor de las clases desvalidas numerosos Congregaciones religiosas de ambos sexos verdaderos ángeles guardianes de nuestros pobres" (p.77). Finalmente, identifica también limitaciones como la falta de "una acción más activa de la Iglesia (en) la educación de las clases populares. Se nota un vacío en materia de escuelas parroquiales, nocturnas para adultos, de escuelas libres para niños, de institutos privados de reeducación de la infancia, de locales de cine educativo" (p.78). 


\section{La Iglesia peruana y la vocación cristiana de promoción del hombre}

La segunda parte de la Carta Pastoral de 1958 empieza reconociendo que "exponer el mal es el comienzo de la salud por lo que es menester buscar medicina apropiada", la misma que debe ser guiada por algunos principios. El principio "primero y fundamental" que se sostiene en la Carta Pastoral es la dignidad de la persona humana basada en su semejanza a Dios: Sólo si nos penetramos profundamente de esta altísima verdad, comprenderemos las consecuencias cristianas y humanas que esta dignidad de la persona humana impone al vivir y al actuar". De ahí nace la exigencia de la libertad y de contar con los medios necesarios. Se clama también contra un materialismo y tecnicismo que hacen perder la visión de la persona humana. Se invoca el esfuerzo personal de vivir el amor, de ayudar con obras sociales, de no vivir en el lujo excesivo provocador. Asimismo las autoridades deben esforzarse por crear medios que despierten, "particularmente en los jóvenes y niños, el deseo de una vida mejor en todo su amplio sentido humano".

Monseñor Dammert en su ponencia sobre las Orientaciones para la vida de la Iglesia en la Primera Semana Social de 1959, hace ver el divorcio entre lo social y lo espiritual y presenta su visión de una Iglesia que denuncia la injusticia y anuncia el Reino de Dios: "Con la mayor tranquilidad o inocencia malgastamos nuestros esfuerzos para procurarnos arbitrios con los cuales aumentar el boato exterior del culto o revestir de plata las andas de alguna imagen de gusto dudoso. En cambio a nuestro alrededor muchos hijos de Dios sufren hambre, padecen enfermedad y miseria. Cuánto bien se haría si reflexionáramos continuamente que la justicia obliga antes que la caridad; que lo recabado por la explotación de nuestros hermanos no se compensa con los donativos, más o menos crecidos, para el culto u obras de caridad. Debemos comprender que el cristianismo coge al hombre integro: no puede disociarse la vida de piedad de los quehaceres cotidianos; no se es buen cristiano, porque se frecuenta los sacramentos, aún diariamente, y no cumple con la justicia social. Corrientemente se confunde a la Iglesia con la Jerarquía eclesiástica, olvidando que en el Cuerpo Místico de Cristo todos los bautizados son sus miembros. Todos tienen la responsabilidad de evangelizar y son solidariamente responsables. Es indispensable recalcar que la doctrina social de la Iglesia brota de sus propias fuentes, que son la justicia y la caridad de Cristo".

En la misma ponencia de orientaciones para la acción de la Iglesia "como Cuerpo Místico de Cristo, no como jerarquía" plantea también la necesidad de enseñar la Doctrina Social de la Iglesia "tal como lo prescriben los Obispos en su Carta Pastoral". Críticamente reconoce que "la falta de clero explica en parte la indiferencia por parte de la Iglesia en difundir los problemas sociales". Por otro lado deja en claro que "la Iglesia es una institución docente, que no debe limitarse a las esferas clericales y religiosas". De igual manera pone énfasis en la misión de los laicos: "intervenir en la elaboración de las estructuras económicas y sociales pero en sentido cristiano y una buena formación doctrinal y competencia".

\section{Epílogo: la Iglesia frente a los de- safíos: los signos de los tiempos y el discernimiento del espíritu.}

Las Semanas Sociales surgieron en Francia a principios del siglo XX (1903) desarrollándose luego en Italia desde 1907 y en América Latina, en Argentina, Brasil, Colombia, México y Uruguay. Jeffrey Klaiber SJ. (1988) da cuenta que la primera Semana Social Nacional en el Perú tomó como modelo a las semanas sociales realizadas en otras partes del mundo y precisa que su organización respondió a la acogida de la Iglesia peruana de las tendencias "modernizantes" y "socializantes" que se habían desarrollando en su interior y que significó "un cambio de rostro de la 
Iglesia" y "una toma de conciencia acerca de los apremiantes problemas sociales". Así también lo ven otros científicos sociales.

Alberto Adrianzén (1990) plantea que desde 1950 se inició en el Perú un nuevo proceso de modernización y desarrollo capitalista, siendo lo más avanzado en la época el movimiento obrero y la apertura de un nuevo espacio para que surjan corrientes liberales y socialistas.

Sinesio López (1991) destaca que en este período un "dato clave del proceso político" ha sido la "conversión ideológica de la Iglesia y de las Fuerzas Armadas, instituciones de enorme gravitación social y política, y su adhesión al campo de las fuerzas reformistas" (p.80).

Julio Cotler (1982) coincide también al señalar que a partir de los años 50 se observa "el desarrollo de una corriente reformista en la Iglesia católica, que buscaba la realización de cambios estructurales en la sociedad civil, a fin de contener el avance del comunismo ateo y fortalecer, en cambio, el carácter cristiano de la sociedad. Los ideólogos de esta corriente emprendieron una campaña de denuncias contra las injusticias existentes en la sociedad peruana, provocando verdaderos escándalos políticos, para luego participar en la organización popular y en las movilizaciones campesinas y de las barriadas..." (p.182).

El documento final de la Primera Semana Social de 1959 ubica a esta en el ambiente general del país, al dejar sentado que "el estudio de la realidad social del país se hace más urgente en el momento actual, en que diversos factores relativos principalmente a la población, economía y cultura general del pueblo del Perú vienen produciendo una trasformación profunda en su composición y en los ideales e intereses de los varios grupos que lo integran. Como consecuencia hay tensiones y problemas de magnitud y naturaleza diferentes de los experimentados en tiempos pasados y particularmente existe un estado de conciencia colectiva que va generalizándose progresivamente, sobre la necesidad de efectuar cambios profundos de la realidad económica y social del país" (p. 223).

La Primera Semana Social tuvo un Comité Organizador conforme al mandato de la Carta Pastoral de 1958 y un Comité Directivo establecido por Decreto Arzobispal del 20 de julio del mismo año. La Semana se realizó entre el $1^{\circ}$ y el 9 de agosto de 1959. Se inscribieron un total de 348 delegados de 106 instituciones de 12 diócesis (Arequipa, Ayacucho, Chiclayo, Huacho, Huancavelica, Huancayo, Huaraz, Lima, Ica, Piura, Tacna y Trujillo), así como de dos Vicariatos (Iquitos, Ucayali) y de una Prelatura (Tarma). La mayor representación la tuvo Lima con 53 instituciones y 216 delegados. Asimis mo, de los 348 delegados, unos 200 eran miembros de la Acción Católica, siendo su participación decisiva a tal punto que hace decir a Klaiber (1988) que "la Semana fue prácticamente un congreso de la Acción Católica" (p.332).

El mismo historiador hace ver que una de las principales contribuciones de la Acción Católica estuvo en el hecho de haber creado en la Iglesia una mayor sensibilidad acerca de la pobreza en el Perú y la necesidad de cerrar la brecha entre las diferentes clases sociales. "Sin duda -afirma Klaiber- su aporte más importante fue la manera en que cambió la propia Iglesia" (p. 331). Sin embargo él mismo hace notar que irónicamente la Primera Semana Social coincidió con el ocaso del movimiento que lo hizo posible. Cabe mencionar entre los miembros más destacados de Acción Católica a César Arróspide, Ernesto Alayza Grundy, Cristóbal de Losada, Gerardo Alarco, Enrique Echegaray. Entre los militantes que empezaron como laicos y terminaron como sacerdotes-asesores figuran José Dammert Bellido, Luis Vallejo Santoni, Eduardo Picher Peña y Gustavo Gutiérrez, quien siendo estudiante de Medicina en 
la Universidad de San Marcos fue presidente del Centro Católico de Barranco $\mathrm{y}$, terminado sus estudios en Europa en 1960, fue asesor arquidiocesano de la Unión de Estudiantes Católicos y luego asesor nacional. Entre los dirigentes que destacaron en provincias están Jorge Polar en Arequipa, Máximo Vega Centeno en Cusco, Edgardo Seoane en Chiclayo. En conjunto, según Klaiber, la Acción Católica Femenina desplegó más dinamismo, sobresaliendo Rebeca Bellido de Dammert, madre del Obispo Dammert, Virginia Candamo de Puente, María Álvarez Calderón de Mujica, Rosina Dugenne de Cebrián y Carolina Elmore de Cobián.

En la Primera Semana Social estuvo también presente el abate Pierre quien, a invitación de Mons. Dammert había llegado al Perú para conocer las barriadas. El abate Pierre alentó a los participantes con su presencia y con sus palabras. A raíz de sus visitas al Perú surgió una rama local de su movimiento conocido como "los traperos de Emaus"

Considerando las expectativas despertadas por la Semana Social y en razón de lo trabajado en la misma, Monseñor Landázuri, a nombre de los obispos del Perú y en su condición de Primado, advirtió en su discurso de clausura que hay que evitar por todos los medios lo que él calificaba de "error funesto", vale decir, el proponer que la Doctrina Social de la Iglesia "debiera ser un programa minucioso, detallado, subordinado a factores políticos o temporales, y aplicable de inmediato a toda especie de problemas. No. Son los pensadores, economistas y estudiosos católicos de los asuntos sociales, en unión de los políticos, empresarios y obreros, quienes están llamados a elaborar un programa de acción concreta pero en base a los grandes principios enseñados por el Magisterio eclesiástico y de acuerdo a las necesidades reales y peculiares circunstancias de tiempo y lugares" (p.251). Al respecto, como lo señalamos al inicio de este trabajo, en la Encíclica Caritas in Veritate el Papa Benedicto XVI nos recuerda que "la Iglesia no tiene soluciones técnicas que ofrecer"(n.9)

Asimismo, en la perspectiva de nuestros días el principio sobre el que gira la doctrina social de la Iglesia es Caritas in Veritate, principio que - afirma el Papa Benedicto XVI- "adquiere forma operativa en criterios orientadores de la acción moral". El Papa nos recuerda dos de estos criterios: "la justicia y el bien común" (n.6).

La Iglesia peruana que convocó a realizar la Primera Social tenía "la aspiración profunda de - en palabras del Presidente del Comité Directivo, Ing. Rómulo A. Ferrero - "buscar los caminos para el establecimiento de un orden social mejor en nuestro país". Ello explica el tema escogido como marco doctrinal: comunidad y bien común. "Amar a alguien - dice el Papa en "Caritas in Veritate" - es querer su bien y trabajar eficazmente por él. Junto al bien individual, hay un bien relacionado con el vivir social de las personas: el bien común" (n.7).

En la perspectiva de la Iglesia peruana de hace cincuenta años atrás, los criterios orientadores de justicia y bien común resumen la lección principal de la Primera Semana Social. Así lo hace ver el P. Felipe Mac Gregor en su exposición que sirvió de marco doctrinal para las ponencias de la Primera Semana: "A procurar el bien común nos obliga, además de nuestra dignidad de hombres la más fundamental exigencia de nuestra fe cristiana. El bien común del Perú, en un sentido muy auténtico, es el mayor bien de cada uno de los peruanos" (p. 27)

Monseñor Dammert sostiene que las orientaciones que se dan en las Semanas Sociales fluyen de dos bases de la doctrina social católica: la dignidad de la persona humana y el principio de la economía al servicio del hombre. "Estos principios provie- 
nen de una fuente propia: la revelación cristiana". Es pertinente recordar una vez más las palabras de Monseñor José Dammert: "Es indispensable recalcar que la doctrina social de la Iglesia brota de sus propias fuentes, que son la justicia y la caridad de Cristo". Una muestra concreta está en el reconocimiento de que "un vasto campo de acción social en todo el Perú para los católicos está en la organización y desarrollo de 'Caritas del Perú'..." (p.175). Además se muestra que en la Arquidiócesis de Lima el Arzobispo Primado ha establecido la "Misión de Lima" "para la ayuda espiritual a los habitantes de las barriadas" (p.176) (23 centros, 136 barriadas con 300 mil habitantes)

La realización y los planteamientos de la Primera Semana Social pueden ser evaluados a través de las siguientes apreciaciones.

En primer lugar, Klaiber (1988) sostiene que el saldo final de la Semana fue una nueva toma de conciencia de la Iglesia acerca de la cuestión social. La Primera Semana Social representó un nuevo comienzo para la Iglesia Peruana porque no se caracterizó por el tono agresivo de los encuentros de la Acción Católica en épocas anteriores, sino más bien por su "positiva apertura hacia toda la sociedad" (p. 352). Monseñor Landázuri, en atención a lo logrado, planteó como conveniente y necesario crear un organismo permanente que "continúe el trabajo de estudio y orientación de esta Primera Semana de Estudios Sociales y que sirva además para preparar las futuras semanas sociales" (Documento Final de la Primera Semana Social. Discurso de clausura)

En segundo lugar, el Presidente del Comité Directivo de la primera Semana, Ing. Rómulo A. Ferrero, declara en su Discurso de apertura que "La aspiración profunda del Episcopado Peruano al promover estas Semanas Sociales es buscar los caminos para el establecimiento de un orden social mejor en nuestro país" (p.14).

En tercer lugar, visto a la distancia de tres décadas posteriores, el Dr. José Rogelio Llerena Quevedo en su discurso pronunciado al culminar la Semana Social del Perú, en 1989, en representación del Consejo Directivo de la referida semana afirma: "Ya la primera Semana Social, en 1959, respondió a una verdadera agitación de los espíritus por comprender integralmente los problemas del país y encontrar orientaciones axiológicas para una ética de la acción política. Por eso, el tema que convocó a más de 350 participantes de por lo menos tres generaciones, fue el de las 'exigencias sociales del catolicismo en el Perú'. En ella, José Luis Bustamante y Rivero, uno de los pocos modelos de consecuencia ética y jurídica de nuestra accidentada vida política, fue capaz de proponer una de las reflexiones más ponderadas del pensamiento peruano sobre 'las clases sociales en el Perú'; Felipe Mac Gregor, planteó entonces cuestiones fundamentales para la concepción misma de todo proyecto nacional que pretenda responder al ethos nuclear de nuestra gente, al tratar 'las nociones de comunidad $y$ de bien común'; César Arróspide, contribuyó a abrir los horizontes de la 'cuestión social' trabajando el tema de 'la promoción cultural' en el Perú; Monseñor Dammert y el Abate Pierre representaron allí una nueva sensibilidad social en la perspectiva de la sensatez cristiana de la vida. Todavía, muchos recordamos con emoción aquellas jornadas que tanto contribuyeron a orientar la inquietud por el Perú" (En Strotmann, 1989 p.259).

Podemos afirmar entonces, teniendo como referente la Primera Semana Social de hace 50 años y la Encíclica Caritas in Veritate que si bien la Iglesia no aporta soluciones técnicas tiene su misión de verdad que cumplir en todo tiempo y circunstancia en favor de la sociedad y de la dignidad del hombre en forma integral. La Primera Semana Social ha sido una forma concreta de realización de la mirada de la Iglesia a los signos de los tiempos y de su fidelidad a la verdad, que, en definitiva, como se deja 
bien sentado en la Caritas in Veritate "es la única garantía de libertad y de la posibilidad de un desarrollo humano integral" (n.9)

\section{REFERENCIAS}

De Lama Angell, Luis Felipe. "Sofocleto" (1958) La tierra prometida. Lima, Juan Mejía Baca. (Premio Nacional de Novela 1958)

Benedicto XVI (2009) Carta Encíclica Caritas in Veritate. Lima, Salesiana

Consejo Nacional de Población (1980) Perú: hechos y cifras demográficas. Lima, Universo.

Cotler, Julio (1979) La crisis política 1930 1968. En: Araníbar, Carlos y otros Nueva Historia General del Perú. Lima, Mosca Azul

Klaiber, Jeffrey S.J. (1988) La Iglesia en el Perú; su historia social desde la Independencia. Lima, PUCP.
López, Sinesio El Dios mortal. Estado, sociedad y política en el Perú del siglo XX. Lima, Instituto Democracia y Socialismo. Gráfica Bellido

Parodi Trece, Carlos (2001) Perú 1960 2000 Políticas económicas y sociales en entornos cambiantes. Lima, Universidad del Pacífico

Primera Semana Social del Perú (1959) Exigencias Sociales del Catolicismo en el Perú. Lima, Secretaria General del Episcopado del Perú

Strotmann, Norberto (editor) (1989) Semana Social del Perú. Documentos de la Semana Social del Perú 1989. Lima, Gráfica San Pablo

Yepes Del Castillo, Ernesto (1979) El desarrollo peruano en las primeras décadas del siglo XX. En: Araníbar, Carlos. y otros op.cit. Lima, Mosca Azul 